\title{
Caracterización del aceite de semilla de borraja extraído con dióxido de carbono supercrítico
}

\author{
Por A. Delgado Andújar *, A. Molero Gómez y E. Martínez de la Ossa \\ Departamento de Ingeniería Química, Tecnología de Alimentos y Tecnologías del Medio Ambiente. \\ Universidad de Cádiz. Apdo. 40. E-11510 - Puerto Real (Cádiz). Spain.
}

\section{RESUMEN}

Caracterización del aceite de semilla de borraja extraído con dióxido de carbono supercrítico.

En el presente trabajo se realiza un estudio comparativo de la calidad del aceite de semilla de borraja obtenido con dióxido de carbono supercrítico y mediante extracción convencional empleando hexano como disolvente. La calidad del aceite extraído con dióxido de carbono es superior. En las condiciones óptimas de operación, los rendimientos encontrados en la extracción con dióxido de carbono, son comparables a los obtenidos en la extracción con hexano, si bien la calidad del aceite es superior.

Por tanto, el proceso de Extracción Supercrítica con dióxido de carbono, al eliminar la etapa de recuperación del disolvente y simplificar sensiblemente el proceso de refinado del aceite, se presenta como una alternativa competitiva con la extracción convencional.

PALABRAS-CLAVE: Aceite de semilla de borraja - Extracción con hexano - Extracción supercrítica - Método (comparación).

\section{SUMMARY}

Characterization of borage seed oil extracted by supercritical carbon dioxide.

The present work is based on a comparative study of the borage seed's oil obtained with supercritical carbon dioxide by means of the conventional extraction using hexane as a solvent. In the optimal working conditions, the results obtained in the extraction with carbon dioxide are comparable to those obtained in the extraction with hexane, although the oil's quality is higher.

Therefore, the process of supercritical extraction with carbon dioxide could be more effective than conventional extraction because the solvent removal is not necessary and several steps of the oil refining process can be reduced notably.

KEY-WORDS: Borage seed oil - Hexane extraction - Method (comparison) - Supercritical extraction.

\section{INTRODUCCIÓN}

Las grasas y aceites han sido empleados para múltiples usos a lo largo de la historia, en iluminación, en cosmética, como lubricantes y principalmente en la alimentación. En este último campo es donde han adquirido una mayor significación, siendo las semillas oleaginosas y las grasas animales las principales fuentes para la producción de aceites y grasas animales (Montiel, 1986).

En los últimos años ha aumentado considerablemente el conocimiento sobre el valor nutricional y los efectos fisiológicos de diversos aceites y grasas. Una muestra clara de estos avances lo constituyen recientes investigaciones llevadas a cabo con el fin de determinar las causas de los efectos beneficiosos encontrados en pacientes que presentaban diversas anomalías, tales como arterioesclerosis, diabetes, trombosis, etc., al serles suministrados ciertos ácidos grasos en la dieta (Williams, 1980; Kumeron, 1975). La gran mayoría de pacientes con estos cuadros clínicos, mostraban dificultades para metabolizar uno de los ácidos grasos esenciales para el organismo, el ácido gamma-linolénico (GLA). Esta deficiencia estaba causada por una alteración de la actividad de la enzima $\Delta-6$ deshidrogenasa, responsable de la formación de GLA a partir del ácido linoléico (Raederstorff, 1992).

Como consecuencia de esta alteración enzimática, se producía un desequilibrio del metabolismo, impidiendo la formación de diversas sustancias, entre las cuales se encuentran las prostaglandinas, responsables de la regulación hormonal de la gran mayoría de funciones que lleva a cabo el organismo (circulatoria, respiratoria, cerebral, etc.).

Consecuentemente, la posibilidad de restaurar adecuadamente los niveles de GLA a través de una dieta suplementaria ha motivado un creciente interés en la búsqueda de aceites ricos en este ácido graso esencial (Carter, 1988; Raederstorff, 1992; Favatti y col, 1991; Syed y col, 1994).

Las principales fuentes comerciales de GLA, son los aceites de semilla de prímula (Oenothera biennis $L$.), borraja (Borago officinalis) y grosella (Ribes nigrum L.). De todos ellos, el que presenta los niveles más altos de GLA es el aceite de semilla de borraja (Fantozzi y col, 1993; Gunstone, 1992), de ahí el interés que suscita.

El aceite de semilla de borraja se extrae convencionalmente empleando disolventes orgánicos, fundamentalmente hexano. Sin embargo en base a evitar posibles cambios en las propiedades físico- 
químicas del GLA; que pudiesen alterar su funcionalidad, estos procesos deben llevarse a cabo bajo condiciones moderadas, prestando especial atención a la temperatura de operación (Eggers, 1985). Además, la especial sensibilización de la sociedad respecto al consumo de productos que ofrezcan las máximas garantías para nuestra salud, así como la creciente concienciación acerca de los riesgos laborales, incentivan la búsqueda de técnicas alternativas que permitan solventar los problemas ocasionados por los métodos convencionales.

Desde esta perspectiva, la extracción con fluidos supercríticos se presenta como una alternativa para complementar o sustituir a los sistemas tradicionales de separación (destilación o extracción con disolventes líquidos), debido a que se consiguen rendimientos energéticos y eficacias de separación mucho mayores (Eggers, 1985).

Actualmente la extracción supercrítica se aplica en gran escala en la descafeinización del café (Sthal, 1990; Bork, 1991), la preparación de extractos de lúpulo para la industria cervecera (Sthal, 1990; Bork, 1991), así como en la producción de extractos y esencias de especias (R.A.P.S., 1993). La gran aplicabilidad mostrada en el campo alimentario radica en gran parte a la utilización de $\mathrm{CO}_{2}$ como disolvente, que a diferencia de los disolventes orgánicos es fácilmente separable del soluto no quedando restos en el producto final, no es tóxico, no inflamable, y es barato y abundante (Subirats y col, 1994). Además, su temperatura crítica $\left(31,1^{\circ} \mathrm{C}\right)$ permite la recuperación de compuestos termolábiles, reduciendo la posibilidad de degradación (Martínez de la Ossa, 1990).

En el presente trabajo se ha llevado a cabo un estudio con el fin de comparar la calidad del aceite de semilla de borraja obtenido con dióxido de carbono supercrítico y mediante dispositivos Soxhlet empleando hexano como disolvente. Con antelación, en un trabajo previo (Delgado, 1999), se establecieron las condiciones operativas óptimas del proceso desde el punto de vista del rendimiento de la Extracción Supercrítica: presión, 300 bar; temperatura, $40^{\circ} \mathrm{C}$; flujo de $\mathrm{CO}_{2}, 1,5 \mathrm{~L} / \mathrm{min}$; tiempo de extracción 3 horas.

\section{MATERIALES Y MÉTODOS}

Los estudios realizados se han llevado a cabo empleando como materia prima semilla de borraja (Borago officinalis).

Debido a las características de la materia prima, se hacía necesaria una etapa previa que permitiese adecuar la semilla al proceso de extracción. Para la molturación de las semillas se hizo uso de un molinillo «Futurmat», modelo «FP», provisto de 18 posiciones de molienda y con una capacidad de carga de $2 \mathrm{Kg}$ El tamizado de las semillas se llevó a cabo empleando un juego de tamices "C.I.S.A», con un tamaño de malla comprendido entre 1,5 y $0,29 \mathrm{~mm}$. La deshidratación de la materia prima, se realizó en una estufa termostatizada "HERAEUS» a una temperatura de $65^{\circ} \mathrm{C}$.

Un diagrama esquemático del equipo utilizado para realizar las extracciones con dióxido de carbono supercrítico, se muestra en la Figura 1, con indicación de los principales elementos que lo constituyen. El protocolo de operación se ha descrito detalladamente en un trabajo previo (Delgado, 1998).

Paralelamente a las extracciones con $\mathrm{CO}_{2}$, se realizaron diversas experiencias de extracción de aceite de semilla de borraja mediante sistema soxhlet empleando hexano como disolvente, durante un tiempo de 16 horas, con el fin de garantizar el agotamiento de la materia prima, y poder realizar de este modo un estudio comparativo entre ambas técnicas de extracción.

La caracterización físico-química de los aceites extraídos se ha llevado a cabo siguiendo las normas que establece la A.O.A.C. (1980) para todos aquellos aceites destinados a consumo. Los parámetros analizados fueron: índice de refracción (refractómetro «ATAGO», modelo 88141; U.N.E. 55-015), densidad (U.N.E. 55-005), viscosidad (viscosímetro de caída de bola tipo Höepler "HAAKE», modelo «B/BH»; U.N.E. 55-105-73) y absorbancia a $270 \mathrm{~nm}$ (espectrofotómetro UV-VIS «SHIMADAZU», modelo «MULTIPURPOSE RECORDING SPECTROPHOTOMETER MPS-2000»; U.N E. 55-047-73). Los distintos índices que se determinaron fueron: acidez (U.N.E. 55011), yodo (U.N.E. 55013), saponificación (U.N.E. 55012) y peróxidos (U.N.E. 55-023-73) e insaponificables (U.N.E. 55-004-73).

La composición en ácidos grasos del aceite extraído se determinó previa metilación de los mismos, siguiendo el método oficial de la AOCS (1996). La identificación y cuantificación de los ácidos grasos se llevó a cabo empleando un cromatógrafo de gases "HEWLETT PACKARD» modelo 5890, provisto de una columna capilar "Carbowax $20 \mathrm{M}$ » y equipado con un detector de ionización de llama (FID).

Todos los productos y reactivos químicos empleados han sido «reactivos para análisis». La pureza del dióxido de carbono utilizado fue del $99,95 \%$ (Carburos Metálicos). 


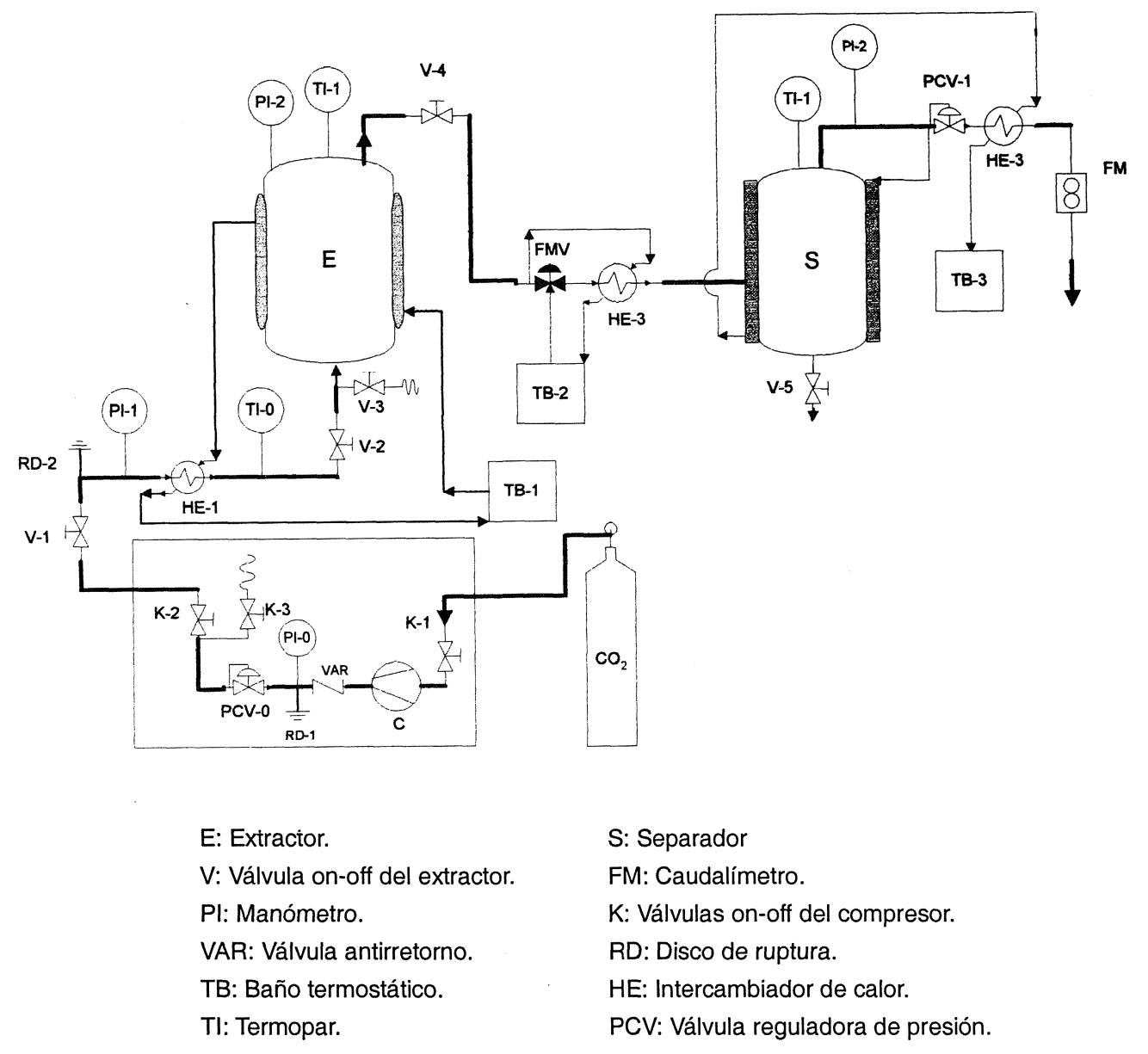

Figura 1

Diagrama esquemático de la Planta de ESC utilizada

\section{DISCUSIÓN DE RESULTADOS}

\subsection{Rendimiento de extracción}

A título comparativo y de cara a la discusión siguiente, en la tabla I se presentan los rendimientos de las extracciones de aceites de diferentes semillas, llevadas a cabo tanto con dióxido de carbono supercrítico como en dispositivos soxhlet utilizando hexano como disolvente.

Tabla I

Comparación de los rendimientos (100 x g aceite/g semilla) de la extracción con $\mathrm{CO}_{2}$-SC y hexano, para diversas semillas vegetales

\begin{tabular}{cccc}
\hline Materia prima & $\mathrm{CO}_{2}$-SC & Hexano & Referencia \\
\hline Semilla de Borraja & 29 & 30,1 & Delgado, 1998 \\
Semilla de Soja & 16,4 & 19,9 & Stahl, 1980 \\
Semilla de Girasol & 36 & 38,4 & Stahl, 1980 \\
Semilla de Colza & 39,3 & 40,1 & Stahl, 1980 \\
Semilla de Uva & 6,9 & 7,5 & Molero, 1995 \\
\hline
\end{tabular}

En un trabajo previo (Delgado, 1998) se establecieron las condiciones óptimas de operación para las extracciones con $\mathrm{CO}_{2}$-SC, tal y como se indicó anteriormente. En estas condiciones, el rendimiento de la extracción fue del $26,4 \%$ frente al $30 \%$ conseguida en la extracción con hexano.

Como se aprecia, los rendimientos en las extracciones con hexano son ligeramente superiores a los encontrados cuando se emplea la ESC como técnica de separación, en todas las materias primas vegetales estudiadas (Stahl, 1980; Molero, 1995; Molero, 1997). Esto es debido, en opinión de todos los autores, a la menor selectividad mostrada por los disolventes líquidos con respecto al dióxido de carbono supercrítico, por lo que además de triglicéridos se extraerán otra serie de compuestos indeseables para el aceite, tales como ácidos grasos libres, fosfolípidos, materia insaponificable, pigmentos, etc. (Stahl, 1980; Friedrich, 1982; Taniguchi, 1986; Zhao, 1997; Delgado, 1998). Este hecho se confirma al analizar los valores de los parámetros resultantes de la caracterización físico-química de los aceites, que se exponen a continuación. 


\section{2. Índices físico-químicos}

En la tabla II se recogen los valores de los índices físico-químicos analizados para los aceites extraídos mediante $\mathrm{CO}_{2}$-SC y con hexano. En referencia a los parámetros físicos analizados, no se encuentran diferencias apreciables entre los dos aceites, a excepción de la viscosidad.

Tabla II

Comparación de los parámetros físico-químicos del aceite de semilla de borraja obtenido con dióxido de carbono supercrítico y hexano

\begin{tabular}{lcc}
\hline \multicolumn{1}{c}{ Parámetros físico-químicos } & $\mathrm{CO}_{2}$-SC & Hexano \\
\hline Indice de refracción & 1,4735 & 1,4702 \\
Densidad $\left(\mathrm{g} / \mathrm{cm}^{3}\right.$ ) & 0,9197 & 0,9052 \\
Viscosidad (cp) & 58,5 & 48,5 \\
Indice de acidez & 11,0 & 20,2 \\
Indice de iodo & 198 & 240 \\
Indice de saponificación & 163 & 180 \\
Insaponificables (\%) & 1,8 & 3,0 \\
Absorb. 270 nm (IgE) & 0,72 & 0,00 \\
Indices de peróxidos & 33,5 & 7,8 \\
\hline
\end{tabular}

Con la finalidad de estudiar el grado de degradación que presentan los aceites extraídos, se analizan tres índices: acidez, peróxidos, y absorción en el UV. El primero de ellos, proporciona información acerca del deterioro hidrolítico que puede haber sufrido el aceite. Por otra parte, el índice de peróxidos trata de evaluar el estado de oxidación inicial de un aceite, así como el deterioro que pueden haber sufrido ciertos componentes, como los tocoferoles (con valor nutritivo y antioxidante) y los polifenoles (antioxidantes). Dada, su poca representatividad sobre el estado global de oxidación de un aceite, este índice se complementa con el valor de la absorción a $270 \mathrm{~nm}$.

- El índice de acidez del aceite extraído con $\mathrm{CO}_{2}$-SC es inferior al encontrado en las extracciones con hexano, debido a que se extraen menos ácidos grasos libres. Estos resultados confirman la mayor selectividad del dióxido de carbono.

- Los bajos valores de índice de peróxido, indican que ambos aceites presentan un grado de oxidación mínimo, siendo muy inferiores a los encontrados para otras materias primas extraídas en las mismas condiciones (Molero, 1995; Molero, 1997). Sin embargo, el aceite obtenido con dióxido de carbono presenta valores algo superiores.
Estudios preliminares (Cocero y col, 1991), indican que la razón de esta inestabilidad podría estribar en la reaccionabilidad del $\mathrm{CO}_{2}$ con algunos compuestos del aceite, facilitando la posterior degradación de tales compuestos por el oxígeno.

- En referencia a la absorción en la región UV, las normas U.N.E. establecen el análisis de los coeficientes de extinción específica a 232 y $270 \mathrm{~nm}$. La primera de ellas, corresponde a una medida de los hidroperóxidos procedentes de la oxidación del ácido linoléico, en tanto que la segunda es indicativa del contenido en $\alpha$-diacetonas y cetonas $\alpha, \beta$-insaturadas (Montiel, 1986). Debido a la mayor estabilidad de estos compuestos con respecto a los hidroperóxidos, la medida a $270 \mathrm{~nm}$ se considera más fiable que la primera, por ello el estudio se centra en el análisis a esta longitud de onda. Los espectros de UV muestran únicamente un pequeño pico a $270 \mathrm{~nm}$ para el aceite extraído con dióxido de carbono, lo que confirma el mínimo grado de degradación que presentan ambos aceites.

El índice de iodo es una expresión cuantitativa del grado de insaturación de una materia grasa. Por ello, y descontando el contenido en ácidos grasos libres, los valores de éste índice dan cuenta de la cantidad de triglicéridos presentes en el aceite. Desde esta perspectiva, la similitud existente entre los valores encontrados, indícan que el contenido de triglicéridos en el aceite es independiente de la técnica de extracción.

Los lípidos saponificables abarcan los triglicéridos, los ácidos grasos libres, las ceras y los fosfolípidos, entre otros. Los resultados obtenidos para este índice son similares para ambos aceites, siendo estos valores muy elevados, lo que refleja la gran cantidad de materia saponificable, en su mayoría triglicéridos, que presenta el aceite de semilla de borraja. La pequeña diferencia estriba en la menor selectividad mostrada por el hexano.

La fracción insaponificable la constituyen básicamente: esteroles, hidrocarburos, tocoferoles y pigmentos (Baudí, 1981). Respecto a este parámetro, sí se aprecian diferencias significativas, en la misma línea que los resultados encontrados por Molero al estudiar la semilla de uva (Molero, 1995), achacables de nuevo, a la menor selectividad del hexano.

\subsection{Composición en ácidos grasos}

En la tabla III se presenta la composición en ácidos grasos del aceite de semilla de borraja obtenido mediante sistema soxhlet empleando hexano como disolvente, así como el extraído con $\mathrm{CO}_{2}-\mathrm{SC}$. 
Tabla III

Composición en ácidos grasos (\%) en los aceites extraídos con $\mathrm{CO}_{2}$ supercrítico, en condiciones óptimas

\begin{tabular}{ccc}
\hline Acidos grasos (\% en peso) & $\mathrm{CO}_{2}$-SC & Hexano \\
\hline Palmítico & 12,94 & 12,64 \\
Palmitoléico & 0,18 & 0,17 \\
Esteárico & 4,45 & 4,81 \\
Oleico & 19,21 & 19,76 \\
Linoléico & 38,43 & 37,13 \\
GLA & 21,65 & 20,02 \\
$\alpha$-linolénico & 0,26 & 0,62 \\
Otros & 2,88 & 4,86 \\
\hline
\end{tabular}

Como se observa, el contenido en ácidos grasos del aceite extraído con $\mathrm{CO}_{2}$ supercrítico es muy similar al encontrado en las extracciones llevadas a cabo con hexano, por lo que no depende de la técnica de extracción. Resultados coincidentes se recogen también en la bibliografía consultada (Fantozzi y col, 1993; Gunstone, 1992).

El elevado contenido del aceite extraído en ácidos grasos insaturados $(86,4 \%)$ frente a la baja proporción de ácidos grasos saturados $(13,6 \%)$, lo hace muy adecuado para su uso en alimentación. Por otra parte, la gran proporción de GLA que presenta, refuerza aún más si cabe el valor nutricional de este aceite.

\section{CONCLUSIONES}

Como se ha demostrado en la discusión precedente, el hecho de que el rendimiento de la extracción del aceite de semilla de borrajas con $\mathrm{CO}_{2}-\mathrm{SC}$ sea ligeramente inferior al conseguido con hexano, lejos de ser inconveniente es significativamente positivo, pues del estudio de los parámetros analizados se concluye lo siguiente:

- El contenido en triglicéridos de ambos aceites es el mismo. No obstante el $\mathrm{CO}_{2}$-SC es más selectivo que el hexano y no extrae otros componentes indeseables que posteriormente habría que eliminar, por lo que la calidad del aceite obtenido es muy superior.

- La composición en ácidos grasos de los dos aceites es idéntica, por lo que, una vez refinados ambos aceites deberían tener las mismas características. Sin embargo, el proceso de refinado del aceite obtenido con $\mathrm{CO}_{2}$-SC será mucho menos intenso que el que nece- sita el obtenido con hexano, dada su mayor calidad.

Por último, hay que tener en cuenta que el empleo de $\mathrm{CO}_{2}$-SC asegura la obtención de un producto final completamente libre de disolvente (que pasa a gas durante la separación por despresurización) cosa que no necesariamente sucede en la destilación de los disolventes orgánicos, y de ahí las cada vez más exigentes restricciones legislativas al respecto, y que esta etapa la más costosa del proceso convencional, tanto más cuanto más disolvente haya que eliminar.

En consecuencia, dado que se elimina la etapa de separación del disolvente por destilación, se asegura la no presencia del disolvente en el aceite extraído y se suaviza el posterior proceso de refinado del aceite, se puede considerar a la extracción con dióxido de carbono supercrítico como una alternativa potencial al proceso convencional.

\section{AGRADECIMIENTOS}

Los autores desean hacer público agradecimiento a la C.I.C.Y.T por la financiación de este trabajo a través del proyecto de $\mathrm{I}+\mathrm{D}$ «Obtención de aceite de semiIla de borrajas y concentrados de ácido gamma-linolénico (GLA) por extracción supercrítica» (ref. QUI97-1080), del que es consecuencia directa el presente trabajo.

\section{BIBLIOGRAFÍA}

A.O.A.C. (Association of Official and Analytical Chemists Society). (1980).— «Standard Methods of Analysis».$13^{\text {th }}$ Ed. Washington D.C.

A.O.C.S. (American Oil Chemical Society).- «Francis' methods".-Official Method, cn- 1- 96.

Baudí, S. (1981).- «Química de los alimentos».Alhambra Mexicana. México.

Bork, M. and Körner, J.P. (1991).—«Extraction by use of supercritical solvents".-Z.F.L. 42 (12), 732-738.

Carter, M.D. (1988). - «Gamma-linolenic acid as a nutrient: Nutritional supplements containing gammalinolenic acid may safely amoliorate certain health problems".-Food Technology, 72-81.

Cocero, M.J. y Calvo, L. (1995)._- «Planta pilotolaboratorio de extracción con $\mathrm{CO}_{2}$ supercrítico para la obtención de aceites de semillas".-Alimentación, Equipos y Tecnología, 67-72.

Delgado, A. (1998).- - Estudio de un proceso de extracción de aceite de semillas de borraja con dióxido de carbono líquido y supercrítico".-Tesis de Licenciatura, Universidad de Cádiz.

Eggers, R. (1985).— «igh pressure extraction of oil seed".-J. Am. Oil Chemists'Soc. 62 (8), 1222-1230.

Fantozzi, P.; Montanari, L.; De Stefano, A. and Sensidoni, A. (1993).—-Gamma-linolenic acid in human nutrition. Sources, extraction process, possible uses".-Atti de II Congresso. I Fluidi Supercritici e Le Loro Applicazioni, 123-140. 
Favatti, F.; King J.W. and Mazzanti, M. (1991).—«Supercritical carbon dioxide extraction of evening primrose oil".- J. Am. Oil Chemists'Soc. 68 (6), 422-427.

Friedrich, J.P. and List, G.R. (1982).- «Characterization of soybean oil extracted by supercritical carbon dioxide and hexane".-J. Agric. Food Chem. 30, 192-193.

Gunstone, F.D. (1992). - «Gamma linolenic acid-ocurrence and physical and chemical properties".-Prog. Lipid Res. 31, 145-161.

Kummerow, F.A. (1975)._- «ipids in atherosclerosis».-J. Food Science, 40, 12-17.

Martínez de la Ossa, E. y Galán Serrano, M.A. (1990).— «Extracción con fluidos supercríticos: (I) Fundamentos».-Ingeniería Química, 169-174.

Molero, A.; Pereyra, C. y Martínez de la Ossa, E. (1995)."Caracterización del aceite de semilla de uva extraído con dióxido de carbono líquido y supercrítico".Grasas y Aceites, 46 (1), 29-34.

Molero, A.; Gordillo, M.D. y Martínez de la Ossa, E. (1997).— «Caracterización del aceite de germen de trigo extraído con dióxido de carbono líquido y supercrítico".-Grasas y Aceites, 48 (2), 87-92.

Montiel, J.A. (1986).—«Estudio de subproductos agrícolas: aceites, proteínas, azúcares y fibra de la semilla y del hollejo de uva palomino".-Tesis Doctoral, Universidad de Cádiz.

Raederstorff, D. and Moser, U. (1992).— «Borage or primrose oil added to standardized diets are equivalent sources for $\gamma$-linolenic acids in rats".-Lipids, 27 (12), 1018-1023.
R.A.P.S. (1993).— «Informationsheet».-Kulmbach, Germany. Stahl, E.; Schutz, E. and Mangold, K. (1980).— «Extraction of seed oils with liquid and supercritical carbon dioxide".-J. Agric. Food Chem. 28, 1153-1157.

Subirats, S.; Millán, L.F. y Blasco, M. (1994)._- «Aplicación de la extracción con fluidos supercríticos en la industria agroalimentaria».-Alimentación, Equipos y Tecnología, 61-64.

Syed Rahmatullah, M.S.K.; Shukla, V.K.S. and Mukherjee, K.D. (1994).- «Enrichment of $\gamma$-linolenic acid concentrates from borage and evening primrose oil fatty acids via lipase-catalyzed esterification".-J. Am. Oil Chemists'Soc. 71 (6), 563-567.

Taniguchi, M.; Kamihira, M.; Tsuji, T. and Kobayashi, T. (1986).— «Application of supercritical $\mathrm{CO}_{2}$ extraction to food processing".-Proc. 3th World Congr. Eng. Tokio, 1040-1043.

Williams, J. (1980)._ «Progrés récents de la recherche sur les acides gras essentiels".-Oléagineux, 35 (10), 457-459.

Zhao, W.; Shisshikura, A.; Fujimoto, K.; Arai, K. and Saito, S. (1987). - «Fractional extraction of rice bran oil with supercritical carbon dioxide".-Agric. Biol. Chem. 51 (7), 1773-1777. 